\title{
Non-Invasive Assessment of Inspiratory Ribcage Muscle Fatigue and Recovery During Two Endurance Tests in Healthy Individuals
}

Thiago Bezerra Wanderley e Lima

Universidade Federal do Rio Grande do Norte

Antonio Sarmento

Universidade Federal do Rio Grande do Norte

Rayane Grayce Silva Vieira

Universidade Federal do Rio Grande do Norte

Esmívany Lhara Freitas Castro

Universidade Federal do Rio Grande do Norte

Francesca Pennati

Politecnico di Milano

Andrea Aliverti

Politecnico di Milano

Vanessa regiane Resqueti

Universidade Federal do Rio Grande do Norte

Guilherme Augusto Freitas Fregonezi ( $\nabla$ fregonezi.guilherme@gmail.com )

Universidade Federal do Rio Grande do Norte

\section{Research Article}

Keywords: Electromyography, respiratory muscles, pletismography, muscle fatigue, tissue oxygenation.

Posted Date: June 23rd, 2021

DOI: https://doi.org/10.21203/rs.3.rs-639412/v1

License: (1) (1) This work is licensed under a Creative Commons Attribution 4.0 International License. Read Full License 


\section{Abstract}

Introduction: Fatigue is defined as a loss in the capacity for developing force and/or velocity of a muscle which is reversible by rest. The aim was to evaluate non-invasively the fatigue and recovery of the inspiratory ribcage muscles during two endurance tests in healthy subjects.

Methods: 22 subjects were evaluated before, during and after performing a respiratory endurance test with normocapnic hyperpnea (NH) and inspiratory pressure threshold load (IPTL). Simultaneous measurements of muscle activity (electromyography), tissue oxygenation (NIRS), pressure (nasal inspiratory pressure), and volume (optoelectronic plethysmography) were performed.

Results: There was a decrease in the maximum relaxation rate (MRR) and increase in the time constant $(\tau)$ after the IPTL test $(p<0.05)$ and a decrease in the peak pressure generated in SNIP after both protocols $(p<0.05)$. Additionally, there was a decrease in shortening velocity and mechanical power only after the IPTL test $(p<0.05)$. The inspiratory ribcage muscles showed a linear drop in the median frequency in the IPTL test and an exponential drop in the $\mathrm{NH}$ test, which was not significant for the development of fatigue and there was a linear increase in the NIRS variables in both protocols.

Conclusion: It was concluded that the inspiratory ribcage muscles undergo changes after performing an endurance test with $\mathrm{NH}$ and IPTL. Additionally, the development of fatigue in these muscles and their consequent changes are more evident in the protocol with IPTL.

\section{Introduction}

Respiratory endurance test (RET) can be performed in different ways and is used to assess the function of respiratory muscles. Normocapnic hyperpnea (NH) and inspiratory pressure threshold load (IPTL) are some of the modalities of RET. NH is performed under conditions of low resistance, high flow and involves the expiratory and inspiratory muscles, while the IPTL is performed in conditions of high resistance, low flow and involves exclusively the inspiratory or expiratory muscles ${ }^{1}$. Due to the difference in their characteristics, these two methods can interfere with the fatigue resistance performance in different ways, leading to different physiological responses².

Fatigue is characterized by a decrease in the muscle capacity to generate strength and/or velocity due to activity $3,4,5$. Respiratory muscle fatigue may limit exercise tolerance and previous reports have suggested that inspiratory muscle fatigue is present in pathologies such as amyotrophic lateral sclerosis, chronic obstructive pulmonary disease (COPD), and heart failure ${ }^{6,7}$. Therefore, it is important to understand the mechanisms involved in the development of fatigue and consequent physiological changes. To assess the development of inspiratory muscle fatigue, the use of non-invasive, less painful, and more accessible assessment options is desirable in clinical practice. These options include surface electromyography (SEMG), muscle parameters of contraction and relaxation, chest wall volumes, and tissue oxygenation. 
The contraction and relaxation parameters of the inspiratory ribcage muscles appear to change during the development of fatigue $e^{5,8,9,10,11}$. Koulouris et al. observed a decrease in the relaxation rate of the inspiratory muscles after a fatigue-induction protocol with an inspiratory resistance valve in healthy subjects $^{12}$. Analysis of these variables can be performed in a simple and non-invasive way by generation of sniff nasal inspiratory pressure (SNIP) with the occlusion of a nostril using a plug 13,14,15,16.

sEMG can be used to evaluate changes in muscle activity and spectrum frequency during the development of peripheral muscle fatigue ${ }^{1,3,7}$. These changes are related to decreased motor unit discharge rates, changes in the shapes of the motor unit action potentials that compose the EMG signal and changes in conduction velocities due to changes in intracellular ph during intense exercise ${ }^{17}$. Studies have shown that development of fatigue induces a change in the power spectrum, with a decrease in the median frequency (MF) as a function of time ${ }^{18,19}$. In addition to the aforementioned analyses, the assessment of lung volumes is important in the study of physiological aspects involved in ventilatory overload and in the study of development of fatigue in the inspiratory ribcage muscles, as reports have suggested that fatigue induction causes decreased operational lung volumes and decreased tidal volume ${ }^{20,21,22}$. Lung volume assessment can be performed in a non-invasive manner using optoelectronic plethysmography (OEP) ${ }^{23,24}$.

In recent years, tissue oxygenation and indirectly blood flow have been studied using the near infrared spectroscopy (NIRS) technique. NIRS is a non-invasive technique that uses the principle of light transmission and absorption to measure the concentrations of oxygenated hemoglobin $\left(\mathrm{O}_{2} \mathrm{Hb}\right)$ and deoxygenated hemoglobin $(\mathrm{HHb})$ in tissues. The sum of these two measures $\left(\mathrm{O}_{2} \mathrm{Hb}+\mathrm{HHb}\right)$ denotes the total hemoglobin concentration ( $\mathrm{tHb}$ ), which is used as a substitute measure for local blood volume in the region of interest ${ }^{25,26}$. From the analysis of these variables, answers can be obtained regarding the tissue oxygenation and inspiratory muscle metaboreflex present in the development of respiratory muscle fatigue.

To the best of our knowledge, the behavior of respiratory muscles during the two types of endurance tests with different characteristics (strength and endurance) and comparison between these modalities have not been clearly analyzed. We hypothesized that development of fatigue in the inspiratory ribcage muscles differs according to RET protocols. We also hypothesized that inspiratory muscle fatigue causes changes in the contraction and relaxation parameters, shortening velocity, and mechanical power of these muscles. Therefore, the main objective of the study was to evaluate fatigue and recovery of the inspiratory ribcage muscles using non-invasive techniques during different endurance tests in healthy individuals.

\section{Materials And Methods}

\section{Study design and participants}


The present crossover study included 22 healthy subjects who voluntarily participated in the study (10 men and 12 women; age: 18-29 years; body mass index [BMl]: 18 to $25 \mathrm{~kg} / \mathrm{m}^{2}$; and no history of smoking and respiratory, cardiac, or neuromuscular diseases). Individuals with forced vital capacity (FVC) and forced expiratory volume in the 1 second $\left(\mathrm{FEV}_{1}\right)<80 \%$ and $\mathrm{FEV}_{1} / \mathrm{FVC}$ ratio $<85 \%$ of the predicted ratio, highly active individuals (assessed by the The International Physical Activity Questionnaire short version-IPAQ) ${ }^{27}$ and those who had nasal congestion or deviated septum were excluded. The study followed the recommendations of the Declaration of Helsinki and was approved by the Research Ethics Committee of Hospital Universitário Onofre Lopes (HUOL-EBSERH/BRASIL) according to protocol number 3.084.956.

\section{Pulmonary function}

Pulmonary function was assessed using the KoKo DigiDoser spirometer (nSpire Health, Inc.; Longmont, CO, USA). $\mathrm{FEV}_{1}, \mathrm{FVC}, \mathrm{FEV} \mathrm{F}_{1} / \mathrm{FVC}$ ratio, and forced expiratory flow between $25-75 \%$ of the FVC maneuver were assessed. The technical procedures followed the recommendations of the American Thoracic Society/European Respiratory Society ${ }^{28}$ and the predicted values were calculated using the reference values for the Brazilian population ${ }^{29}$. Additionally, the slow vital capacity maneuver and the voluntary maximum ventilation test for 15 seconds (MVV) were performed.

\section{Respiratory muscle strength}

Evaluation of respiratory muscle strength was performed using a digital manometer (NEPEBLabCare/UFMG, Belo Horizonte, MG, Brazil) with the participants in a seated position and feet on the floor. The maximum inspiratory pressure (MIP) was obtained from the residual volume and the maximum expiratory pressure (MEP) was obtained from the total lung capacity. To obtain the predicted values, regression equations for healthy Brazilian population were used ${ }^{30}$. Additionally, nasal expiratory pressure (SNEP) and SNIP were also measured. For the SNIP test, previously described equations were used to obtain the reference values ${ }^{31}$.

\section{Sniff curve analysis}

All sniffs were performed at functional residual capacity with the individuals seated on a chair without back support. The contraction and relaxation properties were evaluated from the sniff traces. For the contraction parameters, the contraction time (expressed in $\mathrm{ms}$ ) was calculated as the time to reach the peak pressure ${ }^{11}$, the maximum rate of pressure development (MRPD) was calcultated as the negative peak of the first derivative of the pressure-time curve (MRPD normalized to the sniff peak pressure [MRPD/sniff peak], expressed in $\left.\mathrm{ms}^{-1}\right)^{32}$, and the time to peak shortening (TPS) (expressed in ms) was calcualed as the time to reach MRPD ${ }^{33}$.

For the relaxation parameters, the half relaxation time $(1 / 2 \mathrm{RT})$ (expressed in $\mathrm{ms}$ ) was calculated as the half-time of the relaxation curve, the maximum relaxation rate (MRR) (expressed in $\mathrm{ms}^{-1}$ ) was defined as 
the positive peak of the first derivative of the pressure-time curve (normalized for the peak sniff pressure $)^{15}$. The time constant $(\tau$, tau) of the later monoexponential phase of pressure decay (over 50$70 \%$ of the pressure decay curve) was also calculated ( $y=\exp -\mathrm{t} / \tau)$. The correlation coefficient of the regression line (pressure vs. time) should be greater than 0.98 for the value of $\tau$ to be acceptable. The criteria for selecting appropriate sniffs for further analysis were: (1) sniff performed from functional residual capacity, (2) peak pressure maintained for less than $50 \mathrm{~ms}$, (3) duration of inspiratory effort < $500 \mathrm{~ms}$, and (4) SNIP waveform with a smooth decay curve ${ }^{16}$. The SNIP curve was analyzed using MATLAB software (The MathWorks Inc., Natick, MA, USA).

\section{Chest wall volumes and compartments}

For the assessment of chest wall volumes the OEP system (BTS Bioengineering, Quincy, MA, USA) was used, which included eight photosensitive cameras that captured the movement variation of 89 retroreflective markers placed over predefined regions of the subject's chest and abdomen ${ }^{35}$. Before each measurement, the device was calibrated in static and dynamic ways using a frequency of 60 frames per second to recognize the markers.

The data considered for the OEP analysis included changes in the volume of the chest wall $\left(\Delta \mathrm{V}_{\mathrm{CW}}\right)$, pulmonary ribcage $\left(\Delta \mathrm{V}_{\mathrm{RCp}}\right)$, abdominal ribcage $\left(\Delta \mathrm{V}_{\mathrm{RCa}}\right)$, abdomen $\left(\Delta \mathrm{V}_{\mathrm{AB}}\right)$, and inspiratory time $(\mathrm{Ti})$. Using these values, the shortening velocity index of the inspiratory ribcage $\left(\Delta \mathrm{V}_{\mathrm{RCp}} / \mathrm{Ti}\right)$ and the shortening velocity index of the diaphragm $\left(\Delta \mathrm{V}_{\mathrm{AB}} / \mathrm{Ti}\right)$ were calculated, while the global shortening velocity index of the inspiratory muscles $\left(\Delta \mathrm{V}_{\mathrm{cw}} / \mathrm{Ti}\right)$ was obtained through the sum of these two parameters. In addition, the products of the pressure generated in the SNIP maneuver according to the shortening velocities $\left(\Delta \mathrm{V}_{\mathrm{CW}} / \mathrm{Ti}\right.$, $\Delta \mathrm{V}_{\mathrm{RCp}} / \mathrm{Ti}$, and $\left.\Delta \mathrm{V}_{\mathrm{AB}} / \mathrm{Ti}\right)$ denoted the mechanical power of the global inspiratory muscles $\left(\mathrm{W}_{\text {insp }}\right)$, inspiratory ribcage muscles $\left(\mathrm{W}_{\mathrm{rcm}}\right)$, and diaphragm $\left(\mathrm{W}_{\mathrm{di}}\right)$, respectively ${ }^{34}$. All of these data were analyzed during the SNIP maneuver.

\section{Surface electromyography}

The electromyographic activity of the respiratory muscles was obtained simultaneously with the assessment of chest wall volumes using a trigger. An electromyograph (TeleMyo DTS Desk Receiver ${ }^{\circledR}$; Noraxon USA Inc., Scottsdale, AZ, USA) was used to acquire the signals with 16-bit resolution. The signal was captured at a sampling frequency set at $1500 \mathrm{~Hz}$, with a low-pass filter of $500 \mathrm{~Hz}$, gain of 1000x, and a common-mode rejection index greater than $100 \mathrm{~dB}$. The data were stored in the software MR version 3.8 (Noraxon USA Inc., Scottsdale, AZ, USA). Ag/AgCl bipolar surface electrodes were attached to the skin in the direction of the muscle fibers after the skin was prepared with abrasion, followed by trichotomy and cleaning with $70 \%$ alcohol according to the Surface Electromyography for the Non-Invasive Assessment of Muscles recommendations (SENIAM) ${ }^{35}$. The procedure was performed on the following muscles: 1 ) sternocleidomastoid (SCM), at the lower third of the distance between the mastoid process and the sternoclavicular joint ${ }^{36} ; 2$ ) scalene muscles (ESC), at $5 \mathrm{~cm}$ from the sternoclavicular joint and $2 \mathrm{~cm}$ above 
this point ${ }^{37}$; and 3 ) parasternal (PS) muscle, at the second intercostal space and $3 \mathrm{~cm}$ from the sternum ${ }^{38}$.

\section{SEMG processing and analysis}

SEMG signals were processed using a $20-400 \mathrm{~Hz}$ Butterworth bandpass filter and analyzed in the time and frequency domains to calculate root mean square (RMS) and MF, respectively. During the SNIP maneuvers, each portion of the SEMG signal corresponding to a SNIP maneuver was subjected to RMS and MF analysis. During RET, only the MF was taken into account, which was calculated through continuous wavelet transform technique using Daubechies4 mother in 5-second windows. For analysis, MF and RMS were normalized for each patient by expressing them relative to values obtained at the beginning of the fatigue protocol (i.e., mean of the initial $10 \mathrm{~s}$ ) and plotted as a function of the total time of RET. All sEMG analyses were performed off-line using MATLAB software (The MathWorks Inc., Natick, MA, USA).

\section{Tissue oxygenation}

Assessment of tissue oxygenation was performed using the NIRS device (Portamon; Artinis Medical Systems BV, Elst, Netherlands). The technique is based on the application of light with near-infrared wavelength, considering the principles of absorption and dispersion based on the spatially resolved spectroscopy method ${ }^{25}$. The Portamon is a non-invasive, portable, wireless tool that contains a receiver and three light-emitting diodes spaced at 30,35 , and $40 \mathrm{~mm}$, which capture the absolute concentrations of $\mathrm{O}_{2} \mathrm{Hb}, \mathrm{HHb}$, and $\mathrm{tHb}$. Thus, tissue oxygenation and local blood volume were estimated from these variables. The device uses wavelengths of 760 and $850 \mathrm{~nm}$ and a bluetooth connection that allows online monitoring while the individual performs various activities. Moreover, it does not suffer from interferences in the presence of equipment such as the EMG machine. The equipment was fixed on the subjects' skin over the left SCM using adhesive tapes after the skin was cleaned with $70 \%$ alcohol. It was fixed in a position similar to that described by Shadgan et $\mathrm{al}^{39}$.

\section{Respiratory endurance test}

Two types of respiratory muscle training devices (SpiroTiger, Idiag® ${ }^{\circledR}$, Fehraltorf, Switzerland and POWERbreathe, HaB International Ltd, Southam, UK) were used to perform the NH and the IPTL protocols, respectively. For the $\mathrm{NH}$ test, the parameters were based on a previous study ${ }^{40}$ with the size of the rebreathing bag established at $50 \%$ of the individual's vital capacity, minute volume (VE) adjusted to $70 \%$ of the 15-second MVV, and the respiratory rate (RR) defined according to the manufacturer's recommendations using the formula: Respiratory rate $=A M V /(B a g ~ s i z e ~ \times 1.2)(1 / \mathrm{min})$, where AMV is target ventilation per minute. The MVV level was set at $70 \%$, as previous studies have reported task failure with this type of ventilation ${ }^{41}$. Subjects were asked to breathe holding the pre-determined VE and task failure was defined when the subjects reached volitional exhaustion or when they were unable to maintain RR and VE after three warnings from the evaluator. For the IPTL test, the medium-resistance 
POWERbreathe ${ }^{\circledR}$ Classic (POWERbreathe; HaB International Ltd., Southam, UK) was used and the test was performed with a load of $80 \%$ of the MIP, based on the recommendations of a recent systematic review $^{6}$. Task failure was defined when the subjects reached volitional exhaustion or were unable to overcome the load after 3 warnings from the evaluator, thus ending the test.

\section{Study protocol}

All subjects were previously informed about the study methods and 10 SNIP maneuvers were performed before the beginning of the protocol for the purpose of learning effect. Data collection was performed on two different days separated by a period of 7 days and the order of the devices was randomized using a simple draw with an opaque envelope. On the first day, spirometry and manovacuometry were performed in addition to the initial assessment including filling the assessment form regarding anthropometric data and the level of physical activity. After this stage, a 20-minute rest period was provided before starting the first RET protocol with the selected device. On the second day of the data collection, subjects performed RET only with the second device. During the tests, heart rate $(\mathrm{HR})$ and peripheral arterial saturation $\left(\mathrm{SpO}_{2}\right)$ were monitored in addition to the application of the Borg effort scale before and immediately after RET.

The experimental protocol consisted of the following phases.

Pre-RET phase: subjects were asked to remain in a seated position in a chair without back support, while a single researcher positioned the SEMG electrodes and the retro-reflective OEP markers. Subsequently, the manometer plug was inserted in one of the nostrils, while the contralateral nostril remained unobstructed and the subjects were asked to perform 10 SNIP maneuvers with an interval of 30 seconds between consecutive maneuvers. Subjects were monitored simultaneously in this phase using OEP and SEMG. For each participant, the SNIP maneuver that generated the highest pressure peak was used to analyze the SEMG parameters, OEP parameters, and the parameters obtained from the sniff curve (preRET values).

RET: after the pre-RET phase, subjects remained seated at rest for 15 minutes while information regarding the protocol was provided. RET was performed using the device selected for that day. During RET, simultaneous signals from SEMG and NIRS were acquired. The duration of the test (Tlim) was recorded at the time of task failure according to the aforementioned criteria. Verbal encouragement was provided throughout the protocol.

Recovery phase: after the test, subjects were instructed to immediately remove the nozzle from the used device, place the plug of the manometer in the same nostril that was used previously, and perform 10 SNIP maneuvers with an interval of 30 seconds between consecutive maneuvers. Similar to the protocol in the pre-RET phase, the SEMG signal was captured simultaneously with the OEP. In this phase, all values obtained in the 10 maneuvers were considered for the analysis.

\section{Statistical analysis}


The normality of the data was verified using the Shapiro-Wilk test. In the descriptive analysis, mean and standard deviation were used for the data with normal distribution and median and interquartile range were used for the data with non-normal distribution. For parametric data, the comparisons between the moments and between devices were performed using the two-way repeated-measures analysis of variance (ANOVA). The comparison between the moments of each device (intragroup) was performed separately using the Friedman test in case of nonparametric data or one-way ANOVA in case of parametric data. To avoid type I errors due to multiplicity of post-fatigue moments, correction using twostage false discovery rate test (using a threshold value of $5 \%$ ) was applied in case of statistical significance instead of the post-hoc Bonferroni or Dunn test ${ }^{42}$. The comparison between the devices (intergroup) was performed using independent samples t-test for parametric data and Mann-Whitney $U$ test for nonparametric data. To verify whether the inspiratory ribcage muscles were developing fatigue during the protocols, regression analysis was applied to the MF variable and regression curves adjusted to the maximum values in the sense of the minimum square were used as an index of fatigue development. For all regression analyses, the determination coefficients ( $r 2$ ), slopes, and time constants were calculated during the moments of task failure and recovery (TF and TRec, respectively). For the regression analysis during the recovery period, the starting point at time zero corresponded to the last point of task failure in each muscle. For linear regressions, TF and TRec were calculated as the inverse values of the regression line slope. For nonlinear regressions, the slopes were calculated as the derivatives of the exponential equation at the beginning of the task failure protocol.

Muscle fatigue was confirmed if the following two criteria were met: 1) negative slope in case of linear regressions ${ }^{43}$ and 2 ) decrease to levels below $60 \%$ of the values recorded the beginning of the task failure ${ }^{44}$ in case of exponential regressions. The NIRS variables were monitored in real time and were subsequently analyzed using the Oxysoft software (Artinis Medical Systems BV, Elst, Netherlands). A moving Gaussian filter was applied and linear regression analysis was performed at intervals of $10 \%$ of the total RET duration.

\section{Results}

Altogether, 22 subjects ( 12 men and 10 women) participated in the study. The mean age was $24.36 \pm$ 2.06 years and mean BMI was $22.40 \pm 2.02 \mathrm{~kg} / \mathrm{m}^{2}$. Anthropometric and pulmonary function characteristics are shown in detail in Table 1.

\section{RET parameters}

A significant increase was observed in the scale of perceived exertion $(p<0.001)$ and heart rate $(p<$ $0.001)$ after both protocols, with no significant difference between the protocols. The $\mathrm{NH}$ test tended to have an RET duration longer than the IPTL protocol $(p=0.07)$. The data related to the test parameters, duration, vital signs, and effort scale are presented in Table 2.

\section{Respiratory endurance test}




\section{Median frequency}

The inspiratory ribcage muscles showed a linear drop in the MF only during the IPTL protocol, with SCM exhibiting the highest slope $(-0.073 \mathrm{r} 2=0.649)$. During the $\mathrm{NH}$ protocol, there was an exponential decrease in the MF of most of the muscles except SCM, which exhibited a linear drop with a slope of $-0.062 \mathrm{r} 2=0.779$. The inspiratory ribcage muscles showed a decay constant of $17.37 \mathrm{~s}$ in the IPTL protocol and $18.36 \mathrm{~s}$ in the $\mathrm{NH}$ protocol. In both the tests, SCM exhibited the lowest time constant value $(\mathrm{TF}=13.54 \mathrm{~s}$ in IPTL and TF $=15.88 \mathrm{~s}$ in $\mathrm{NH})($ Figure 1$)$.

\section{Tissue oxygenation}

Both the protocols resulted in similar behaviors in terms of the variables related to tissue oxygenation. There was a linear increase in all the analyzed variables. In both the protocols, $\mathrm{tHb}$ exhibited the highest slope values $\left(0.209 \mathrm{r} 2=0.974\right.$ and $0.116 \mathrm{r} 2=0.924$ in IPTL and $\mathrm{NH}$, respectively). $\mathrm{O}_{2} \mathrm{Hb}$ exhibited the lowest slope values $(0.092 \mathrm{r} 2=0.938$ and $0.057 \mathrm{r} 2=0.550$ in IPTL and $\mathrm{NH}$, respectively) (Figure 2).

\section{Recovery phase}

\section{Contractile and relaxation parameters}

The relaxation parameters obtained from the sniff traces exhibited a statistically significant difference only in the intragroup comparison in the IPTL test. MRR showed a statistically significant decrease from the first to the sixth post-RET maneuver $(p<0.05)$ and the time constant $(\tau)$ increased significantly from the first to the fourth maneuver $(p<0.01)$, returning to baseline values in the tenth maneuver (Figure 3 ).

The peak pressure values generated during all SNIP maneuvers after RET were lower than the pre-RET values in both the protocols in the intragroup comparison $(p<0.05)$ (Figure 4).

\section{Shortening velocity index and mechanical power}

In the intragroup comparison of the shortening velocity indices in the IPTL test, there was a decrease in $\Delta \mathrm{V}_{\mathrm{RCp}} / \mathrm{Ti}$ from the second to the fifth post-RET maneuver $(\mathrm{p}<0.05) . \Delta \mathrm{V}_{\mathrm{CW}} / \mathrm{Ti}$ and $\Delta \mathrm{V}_{\mathrm{AB}} / \mathrm{Ti}$ did not exhibit any significant changes in the post-RET maneuvers when compared with the pre-RET values. In the $\mathrm{NH}$ test, no significant changes were observed in any of these variables in the intragroup comparison as well as in the intergroup comparison (Figure 5).

In the intragroup comparison of mechanical power, a decrease in $\mathrm{W}_{\text {insp }}$ was observed from the second to the fifth maneuver in the IPTL test $(p<0.05)$. Additionally, there was a decrease in $W_{r c m}$ from the first to the eighth maneuver $(p<0.05)$. $W_{d i}$ did not show any significant changes. In the $\mathrm{NH}$ test, there were no significant changes in any of the variables in the intragroup comparison as well as in the intergroup comparison (Figure 6).

\section{Muscle electrical activity}


Similar muscle activation behaviors were observed in SCM, ESC, and PS muscles in both the tests, with a significant decrease $(p<0.05)$ in the \% RMS in all post-RET moments when compared with the pre-RET values in the intragroup comparison. In the intergroup comparison, SCM showed a statistically significant decrease in the electrical activity in the IPTL test when compared with the $\mathrm{NH}$ test from the second to the fourth maneuver $(p<0.05)$ (Figure 7). In the analysis of the recovery phase of the MF, a Trec of $19.98 \mathrm{~s}$ was observed in the IPTL test and a Trec of $15.82 \mathrm{~s}$ was observed in the NH test. SCM exhibited a longer recovery time than other muscles in both the tests $($ Trec $=20.73 \mathrm{~s}$ in IPTL and Trec $=17.37 \mathrm{~s}$ in NH) (Figure 1).

\section{Discussion}

\section{Main findings}

An IPTL protocol with $80 \%$ of the MIP resulted in a linear decrease in the MF of the inspiratory ribcage muscles, with a consequent decrease in the muscle relaxation properties (MRR and tau), peak pressure, muscle shortening velocity, and mechanical power of the inspiratory ribcage muscles. Both RET protocols caused an increase in the concentrations of $\mathrm{O}_{2} \mathrm{Hb}, \mathrm{HHb}$, and $\mathrm{tHb}$ in the SCM muscle during the test.

\section{Inspiratory ribcage muscle fatigue}

Inspiratory muscle fatigue has been assessed in a variety of non-invasive ways including measurement of the duration for which an individual is able to sustain a load or volume, measurement of maximum respiratory pressures before and after fatigue induction, and reporting the adverse sensations such as dyspnea and scale of effort ${ }^{6}$. Due to the subjectivity and lack of precision of these analyses, SEMG has been used to analyze the development of muscle fatigue by considering the frequency parameters ${ }^{45}$. Studies have shown that the MF shows a decrease as a function of time during the fatigue protocol (dynamic or isometric) and one of the ways to perform this evaluation is to analyze the slope generated during a particular protocol ${ }^{46,47,48}$.

After analyzing each muscle individually, we observed that SCM exhibited the greatest negative slope. This finding may be related to the fact that SCM has a higher proportion of type II fibers $(65 \%)$ when compared with ESC and PS (39\% and $38 \%$, respectively) ${ }^{49,50}$. It may also be related to the observations that SCM had a shorter TF and a higher Trec than the other muscles in both protocols, and it was the only muscle that showed a linear decrease during the $\mathrm{NH}$ protocol.

During the $\mathrm{NH}$ protocol, an exponential decrease was observed in the MF of the inspiratory ribcage muscles, but it did not reach the lower limit used to characterize fatigue (60\%). Previous studies assessing the development of inspiratory muscle fatigue during the $\mathrm{NH}$ protocol analyzed diaphragm fatigue using invasive techniques ${ }^{1,40,51,52}$. In one of these studies, Renggli et al. included healthy subjects who underwent an RET protocol with $\mathrm{NH}$. The authors observed that diaphragm and abdominal muscle fatigue occurred at the beginning of the test, even before task failure, and the recruitment of inspiratory 
and expiratory muscles in the chest wall was observed to maintain high ventilatory levels ${ }^{40}$. The RET time in their study was higher than that in the present study. Therefore, the recruitment of expiratory muscles and a shorter RET duration may be related to the finding that the $\mathrm{NH}$ protocol did not reach significant levels for the development of fatigue.

\section{Tissue oxygenation during RET}

Studies have shown that the presence of inspiratory muscle fatigue increases sympathetic nerve activity, as this response is mediated mainly by type III and IV afferent fibers from the diaphragm ${ }^{53,7}$. Therefore, there is a compromised blood flow to the inactive muscles and redirection of blood flow to the active muscles. This process is called inspiratory muscle metaboreflex. Shadgan et al. and Basoudan et al. observed an increase in $\Delta \mathrm{HHb}, \Delta \mathrm{tHb}$, and no change in $\Delta \mathrm{O}_{2} \mathrm{Hb}$ during a protocol with inspiratory resistive load. Guenette et al. observed an increase in the blood flow to the respiratory muscles with an increase in the ventilatory work during the $\mathrm{NH}$ protocol ${ }^{39,54,55}$. Our findings were similar to the findings of these studies, in which there was an increase in $\Delta \mathrm{tHb}$ as a function of time, and this appears to be due to a redirection of blood flow from inactive muscles to respiratory muscles during activity. However, in the present study, the local blood flow of the peripheral muscle was not estimated. In addition, there was an increase in $\Delta \mathrm{HHb}$ without a decrease in $\Delta \mathrm{O}_{2} \mathrm{Hb}$ in both the protocols. This increase in $\Delta \mathrm{HHb}$ can be explained by the Bohr effect, which results in oxygen discharge due to the acidity resulting from the activity and an increase in temperature. Regardless of the protocol used, an increase in respiratory work resulted in changes in blood flow and tissue oxygenation.

\section{Recovery phase}

\section{Contractile and relaxation parameters}

Previous studies have shown that the SNIP maneuver has a high correlation with esophageal pressure, as it is related to the pressure in the diaphragm and also associated with other inspiratory muscles ${ }^{14}$. Previous reports have suggested that fatigue causes changes in the relaxation properties such as MRR and $\tau^{10,14,15}$. Mador et al. conducted a study involving healthy subjects and observed that after inducing fatigue by imposing an inspiratory load, there was a decrease in MRR and an increase in the time constant $\tau^{15}$. In the present study, we observed a decrease in MRR and an increase in the time constant $\tau$ after RET with IPTL and the values returned near the baseline values in the seventh and in the tenth maneuvers, respectively. This result may be related to changes in the sarcoplasmic reticulum due to the presence of fatigue, resulting in changes in the calcium $\left(\mathrm{Ca}^{2+}\right)$ release and in the uptake capacity. Additionally, the accumulation of phosphorus $\left(\mathrm{P}_{\mathrm{i}}\right)$ during the development of fatigue can alter the maximum rate of muscle relaxation $56,57,58,59$.

\section{Shortening velocity index and mechanical power}


We observed that there was a decrease in the shortening velocity $\left(\Delta \mathrm{V}_{\mathrm{RCp}}\right)$ and in the mechanical power $\left(\mathrm{W}_{\text {insp }}\right.$ and $\left.\mathrm{W}_{\text {rcm }}\right)$ only after the IPTL protocol. Since muscle fatigue causes a decrease in strength, velocity and power, the results observed only in the IPTL protocol are related to the fact that the MF reached the levels of characterization for the presence of fatigue only during the IPTL protocol. These changes occur due to metabolic changes induced by fatigue that can affect cross-bridges and actinmyosin binding, preventing the formation of a new coupling-uncoupling cycle and reducing muscle contraction $^{60}$.

\section{Muscle electrical activity}

The electrical activity of inspiratory ribcage muscles in both protocols was similar in the intragroup comparison, with a decrease in the \% RMS in the post-RET maneuvers when compared with the values from the pre-RET maneuvers. In the intergroup comparison, there was a significant difference in the electrical activity of SCM, with a greater percentage drop in the IPTL test. This finding may be related to the pattern of muscle recruitment during RET. For example, SCM had a more negative inclination in the IPTL protocol, which may be related to the fact that recruitment of SCM was greater in this protocol, potentiating the development of fatigue. A decrease in the muscle electrical activity resulting from increased respiratory effort may be related to a decrease in the conduction velocity of the action potential, consequently decreasing the electrical excitation of the muscle $e^{61,62}$.

\section{Strengths and limitations of the study}

To the best of our knowledge, this is the first study to analyze the physiological behavior of respiratory muscles before, during, and after performing different respiratory endurance tests using a variety of instruments and assessments such as OEP, SEMG and NIRS. Inspiratory muscle fatigue is present in several pathologies such as neuromuscular diseases and COPD. Understanding the physiological changes caused by the development of fatigue in inspiratory ribcage muscles during these different RET becomes important to have a response when it comes to the best method to assess fatigue in patients with low resistance.

The present study has some limitations. A high percentage of MIP in the IPTL protocol may have led to lower duration of the test, limiting the potentiation of the development of fatigue. We did not use another NIRS probe for the assessment of peripheral muscle oxygenation in order to evidence the inspiratory metaboreflex and analysis of the rectus abdominis SEMG was not performed. Hence, questions regarding the differences in its recruitment between the protocols could not be elucidated.

\section{Conclusion}

The behavior of the inspiratory ribcage muscles differs between the NH protocol and the IPTL protocol of the endurance test. Changes in the relaxation properties (MRR and $\tau$ ), shortening velocity, and mechanical power were observed only after testing with the IPTL protocol. Development of fatigue in the inspiratory ribcage muscles and consequent changes are more evident in the IPTL protocol, which seems to recruit 
more inspiratory ribcage muscles. In general, inspiratory muscle fatigue, which is observed in several pathologies, causes significant changes in the behavior of the inspiratory ribcage muscles.

\section{Declarations}

\section{Ethical approval}

The study was conducted in accordance with the guidelines of the Declaration of Helsinki, and approved by the ethics committee of the Hospital Universitário Onofre Lopes (HUOL/EBSERH - Brazil) under number 3.084.956.

\section{Consent to participate}

Informed consent was obtained from all individual participants included in the study.

\section{Funding}

This study were financed in part by the Coordenação de Aperfeiçoamento de Pessoal de Nível Superior Brasil (CAPES) Finance Code 001 and Project PROCAD CAPES 2013 number 88881.068409/2014-01. Vanessa Regiane Resqueti is a fellow of the Conselho Nacional de Desenvolvimento Científico e Tecnológico (CNPq) - process number 315580/2018-6. Guilherme Augusto de Freitas Fregonezi is fellow of the Conselho Nacional de Desenvolvimento Científico e Tecnológico (CNPq) - process number 312876/2018-1.

\section{Conflicts of interest/Competing interests}

The authors declare that they have no competing interests.

\section{Availability of data and material}

The data that support the findings of this study and additional information are available from the corresponding author upon request.

\section{Author contributions}

TBWL, AS, and GAFF designed the research. TBWL, ELFC, RGSV, VR and GAFF collected data. FP developed the software for EMG and pressure data analysis. TBWL, AS, GAFF, FP and AA analyzed and interpreted the data, as well as drafted the manuscript. All authors reviewed the manuscript drafts and inputted corrections. All authors approved the final version of the manuscript. Disclosures The authors declare no competing interests.

\section{References}


1. Kabitz H.J., Walker D.J., Schwoerer A., Schlager D., Walterspacher S., Storre J.H., et al. Biometric approximation of diaphragmatic contractility during sustained hyperpnea. Respir Physiol Neurobiol. 176,90-7. https://doi.org/10.1016/j.resp.2011.01.011(2011)

2. Verges S., Renggli A.S., Notter D.A., Spengler C.M. Effects of different respiratory muscle training regimes on fatigue-related variables during volitional hyperpnoea. Respir Physiol Neurobiol. 169,282-90. https://doi.org/10.1016/j.resp.2009.09.005 (2009)

3. Laghi F., Topeli A., Tobin M.J. Does resistive loading decrease diaphragmatic contractility before task failure? J App/ Physiol. 85,1103-12. https://doi.org/10.1152/jappl.1998.85.3.1103 (1998)

4. Walker D.J., Farquharson F., Klenze H., Walterspacher S., Storz L., Duerschmied D, et al. Diaphragmatic fatigue during inspiratory muscle loading in normoxia and hypoxia. Respir Physiol Neurobiol. 227,1-8, 20. https://doi.org/10.1016/j.resp.2016.01.006 (2016)

5. Gibson G.J., Whitelaw W., Siafakas N., Supinski G.S., Fitting J.W., Bellemare F., et al. ATS/ERS Statement on respiratory muscle testing. Am J Respir Crit Care Med. 166, 518-624. https://doi.org/10.1164/rccm.166.4.518 (2002)

6. Janssens L., Brumagne S., Mcconnell A.K., Raymaekers J., Goossens N., Gayan-ramirez G. The assessment of inspiratory muscle fatigue in healthy individuals: A systematic review. https://doi.org/10.1016/j.rmed.2012.11.019 (2013)

7. Katayama K., Iwamoto E., Ishida K., Koike T., Saito M. Inspiratory muscle fatigue increases sympathetic vasomotor outflow and blood pressure during submaximal exercise. Am J Physiol. 302,1167-75. https://doi.org/10.1152/ajpregu.00006.2012 (2012)

8. Jewell B.R., Wilkie D.R. The mechanical properties of relaxing muscle. J Physiol. 152,30-47. https://doi.org/10.1113/jphysiol.1960.sp006467 (1980)

9. Edwards BYRHT, Hill D.K., Jones D.A. Metabolic changes associated with the slowing of relaxation fatigued mouse muscle. J Physiol. 251,287-301. https://doi.org/10.1113/jphysiol.1975.sp011093 (1975)

10. Kyroussis D., Mills G., Hamnegard C.H, Road J., Green M., Wragg S., et al. Inspiratory muscle relaxation rate assessed from sniff nasal pressure. Thorax. 49,1127-33. https://doi.org/10.1136/thx.49.11.1127 (1994)

11. Romer L.M., McConnell A.K. Inter-test reliability for non-invasive measures of respiratory muscle function in healthy humans. Eur J App/ Physiol. 91,167-76. https://doi.org/10.1007/s00421-0030984-2 (2004)

12. Koulouris N., Vianna L.G., Mulvey D.A., Green M., Moxham J. Maximal relaxation rates of esophageal, nose, and mouth pressures during a sniff reflect inspiratory muscle fatigue. Am Rev Respir Dis. 139,1213-7. https://doi.org/10.1164/ajrccm/139.5.1213 (1989)

13. Dassios T.G., Doudounakis S., Dimitriou G. Maximum Rate of Pressure Development and Maximal Relaxation Rate of Respiratory Muscles in Patients with Cystic Fibrosis. Respir Care. 58,474-81. https://doi.org/10.4187/respcare.01930 (2013) 
14. Esau S.A., Bellemare F., Grassino A., Permutt S., Roussos C., Pardy R.L. Changes in relaxation rate with diaphragmatic fatigue in humans. J Appl Physiol. 54,1353-60.

https://doi.org/10.1152/jappl.1983.54.5.1353 (1983)

15. Mador M.J, Kufel T.J Effect of inspiratory muscle fatigue on inspiratory muscle relaxation rates in healthy subjects. Chest. 102,1767-73. https://doi.org/10.1378/chest.102.6.1767 (1992)

16. Sarmento A., Aliverti A., Marques L., Pennati F., Dourado-Júnior M.E., Fregonezi G., et al. Multiparametric analysis of sniffnasal inspiratory pressure test in middle stage amyotrophic lateral sclerosis. Front Neurol. 2, 9:306 https://doi.org/10.3389/fneur.2018.00306 (2018)

17. Beck T.W., Stock M.S., De Freitas J.M. Shifts in EMG spectral power during fatiguing dynamic contractions. Muscle Nerve. 50,95-102. https://doi.org/ 10.1002/mus.24098 (2014)

18. de Luca C.J. The use of surface electromyography in biomechanics. J Appl Biomech. 13,135-63. (1997)

19. Gallagher C.G., Hof V.I., Younes M. Effect of inspiratory muscle fatigue on breathing pattern. J Appl Physiol. 59, 1152-8. https://doi.org/10.1152/jappl.1985.59.4.1152 (1985)

20. Verges S., Notter D., Splenger C.M. Influence of diaphragm and rib cage muscle fatigue on breathing during endurance exercise. Respir Physiol Neurobiol. 154, 431-42. https://doi.org/10.1016/j.resp.2005.12.007 (2005)

21. Illi S.K., Hostettler S., Aliverti A., Spengler C.M. Compartmental chest wall volume changes during volitional hyperpnoea with constant tidal volume in healthy individuals. Resp Physiol Neurobio./ 185, 410-5. https://doi.org/10.1016/j.resp.2012.08.018 (2013)

22. Hostettler S., Illi S.K., Mohler E., Aliverti A., Spengler C.M. Chest wall volume changes during inspiratory loaded breathing. Resp Physiol Neurobiol. 175,130-9.

https://doi.org/10.1016/j.resp.2010.10.001 (2011)

23. Aliverti A., Ghidoli G., Dellaca R.L., Pedotti A., Macklem P.T. Chest wall kinematic determinants of diaphragm length by optoelectronic plethysmography and ultrasonography. J Appl Physiol. 94 621630. https://doi.org/10.1152/japplphysiol.00329.2002 (2003)

24. Aliverti A., landelli I., Duranti R., Cala S.J., Kayser B., Kelly S., Misuri G., Pedotti A., Scano G., Sliwinski P., Yan S., Macklem P.T. Respiratory muscle dynamics and control during exercise with externally imposed expiratory flow limitation. J Appl Physiol. 92 1953-1963.

https://doi.org/10.1152/japplphysiol.01222.2000 (2002)

25. Adami A., Cao R., Porszasz J., Casaburi R., Rossiter H.B. Reproducibility of NIRS assessment of muscle oxidative capacity in smokers with and without COPD. Respir Physiol Neurobiol. 235,18-26. https://doi.org/ doi:10.1016/j.resp.2016.09.008 (2017)

26. Tanaka T., Basoudan N., Melo L.T., Wickerson L., Brochard L.J., Goligher E.C., et al. Deoxygenation of inspiratory muscles during cycling, hyperpnoea and loaded breathing in health and disease: a systematic review. Clin Physiol Funct Imaging. 38, 554-65. https://doi.org/10.1111/cpf.12473 (2017) 
27. Pardini, R., Matsudo, S.M.,Araújo, T., Matsudo, V., Andrade, E., Braggion, G., Andrade, D., Oliveira, L., Figueira, Jr. A., Raso, V. Validação do questionário internacional de nível de atividade física (IPAQ versão 6): estudo piloto em adultos jovens brasileiros. Rev. Bras. Ciên. (2001)

28. Miller M.R., Hankinson J., Brusasco V., Burgos F., Casaburi R., Coates A., Crapo R., Enright P. et al. J ATS/ERS Task Force. Standardisation of spirometry. Eur Respir J. 26,319-38. https://doi.org/10.1183/09031936.05.00034805 (2005)

29. Pereira C.A, Sato T., Rodrigues S.C. Novos valores de referência para espirometria forçada em brasileiros adultos de raça branca. Jornal Brasileiro de Pneumologia 33,97-406. https://doi.org/10.1590/S1806-37132007000400008 (2007)

30. Neder J.A., Andreoni S., Lerario M.C., Nery L.E. Reference values for lung function tests. II. Maximal respiratory pressures and voluntary ventilation. Brazilian journal of medical and biological research. 32,719-27. https://doi.org/10.1590/s0100-879x1999000600007 (1999)

31. Araújo P.R.S., Resqueti V.R., Nascimento Jr. J., et al. Valores de referência da pressão inspiratória nasal em indivíduos saudáveis no Brasil: estudo multicêntrico. Jornal Brasileiro de Pneumologia. 38,700-7. https://doi.org/10.1590/S1806-37132012000600004 (2012)

32. Metzger J.M., Moss R.L. Shortening velocity in skinned single muscle fibers. Influence of filament lattice spacing. Biophys J. 52,127-31. https://doi.org/10.1016/S0006-3495(87)83197-1 (1987)

33. Herve P., Lecarpentier Y., Brenot F., Clergue M., Chemla D., Duroux P. Relaxation of the diaphragm muscle: influence of ryanodine and fatigue. J Appl Physiol. 65,950-6. https://doi.org/10.1152/jappl.1988.65.5.1950 (1985)

34. Aliverti A., Cala S.J., Duranti R., Ferrigno G., Kenyon C.M., Pedotti A., et al. Human respiratory muscle actions and control during exercise. J Appl Physiol. 83,1256-69. https://doi.org/ 10.1152/jappl.1997.83.4.1256 (1997)

35. Hermens H.J., Freriks B., Disselhorst-Klug C.R.G. Development of recommendations for SEMG sensors and sensor placement procedures. J Electromyogr Kinesiol. 10:361-74. https://doi.org/10.1016/S1050-6411(00)00027-4 (200)

36. De Andrade A.D., Silva T.N., Vasconcelos H., Marcelino M., Rodrigues-Machado M.G., Filho V.C., Moraes N.H., Marinho P.E A.C. Inspiratory muscular activation during threshold therapy in elderly healthy and patients with COPD. J Electromyogr Kinesiol. 15,631-9. https://doi.org/10.1016/j.jelekin.2005.06.002 (2005)

37. Da Cunha A.P.N.M. Silva, T.N.S., França, E.R.T., Amorim, C., Galindo Filho, V.C., De Andrade A.D. Efeito do alongamento sobre a atividade dos músculos inspiratórios na DPOC. 7:6. (2005).

38. Duiverman M.L., van Eykern L.A., Vennik P.W., Koeter G.H., Maarsingh E.J., Wijkstra P.J. Reproducibility and responsiveness of a noninvasive EMG technique of the respiratory muscles in COPD patients and in healthy subjects. J Appl Physiol. 96,1723-9. https://doi.org/10.1152/japplphysiol.00914.2003 (2004)

39. Shadgan B., Guenette J.A., Sheel A.W., Reid W.D. Sternocleidomastoid muscle deoxygenation in response to incremental inspiratory threshold loading measured by near infrared spectroscopy. 
Respir Physiol Neurobiol. 178,202-9. https://doi.org/ 10.10.16/j.resp.2011.06.001 (2011)

40. Renggli A.S., Verges S., Notter D.A., Spengler C.M. Development of respiratory muscle contractile fatigue in the course of hyperpnoea. Respir Physiol Neurobiol. 164,366-72. https://doi.org/10.1016/j.resp.2008.08.008 (2008)

41. Verges S., Renggli A.S., Notter D.A., Spengler C.M. Effects of different respiratory muscle training regimes on fatigue-related variables during volitional hyperpnoea. Respir Physiol Neurobiol. 169,282-90. https://doi.org/10.1016/j.resp.2009.09.005 (2009)

42. Benjamini Y., Krieger A.M., and Yekutieli D. Adaptive linear step-up procedures that control the false discovery rate. Biometrika. 93,491-507. (2006)

43. Cifrek M., Tonković S., and Medved V. Measurement and analysis of surface myoelectric signals during fatigued cyclic dynamic contractions. Measurement.. 27,85-92. https://doi.org/10.1016/S0263-2241(99)00059-7 (2000)

44. Aubier M., Farkas G., De Troyer A., Mozes R., and Roussos C. Detection of diaphragmatic fatigue in man by phrenic stimulation. J Appl Physiol. 50,538-544. https://doi.org/10.1152/jappl.1981.50.3.538 (1981)

45. Grassino A., M.D., Macklem, P.T. Respiratory muscle fatigue and ventilatory failure. Chest. 97,89-96. https://doi.org/10.1378/chest.97.3_Supplement.89S (1990)

46. Gertler J., Cao J. EMG spectral power and fatigue. Wiley Intersci. 50,1-15. (2004)

47. Merletti R., Knaflitz M., De Luca C.J. Myoelectric manifestations of fatigue in voluntary and electrically elicited contractions. J Appl Physiol. 69,1810-20. https://doi.org/10.1152/jappl.1990.69.5.1810 (1990)

48. Komi P. V., Tesch P., Komi V., Tesch P. EMG Frequency Spectrum, Muscle Structure and Fatigue. Eur J Appl Physiol. 50,41-50. https://doi.org/10.1007/bf00421103 (1979)

49. Johnson M.A., Polgar J., Weightman D., and Appleton D. Data on the distribution of fibre types in thirty-six human muscles. An autopsy study. Journal of the neurological sciences. 18,111-129. https://doi.org/10.1016/0022-510X(73)90023-3 (1973)

50. Vikne H., Gundersen K., Liestol K., Maelen J., and Vollestad N. Intermuscular relationship of human muscle fiber type proportions: slow leg muscles predict slow neck muscles. Muscle \& nerve. 45,527535. https://doi.org/10.1002/mus.22315 (2012)

51. Bai T.R., Rabinovitch B.J., Pardy R.L. Near-maximal voluntary hyperpnea and ventilatory muscle function. J Appl Physiol Respir Environ Exerc Physiol. 57,1742-8. https://doi.org/10.1152/jappl.1984.57.6.1742 (1984)

52. Verges S., Bachasson D., Wuyam B. Effect of acute hypoxia on respiratory muscle fatigue in healthy human. Respir Res. 11,109. https://doi.org/10.1186/1465-9921-11-109 (2010)

53. Sheel A.W., Boushel R., Dempsey J.A. Competition for blood flow distribution between respiratory and locomotor muscles: Implications for muscle fatigue. J Appl Physiol. 125,820-31. https://doi.org/10.1152/japplphysiol.00189.2018 (2018) 
54. Guenette J.A., Vogiatzis I., Zakynthinos S., et al. Human respiratory muscle blood flow measured by near-infrared spectroscopy and indocyanine green. J Appl Physiol. 104,1202-1210. https://doi.org/10.1152/japplphysiol.01.160.2007 (2008)

55. Basoudan N., Shadgan B., Guenette J.A., Road J., Reid W.D. Effect of acute hypoxia on inspiratory muscle oxygenation during incremental inspiratory load in healthy adults. Eur J Appl Physiol. 116,841-850. https://doi.org/10.1007/s00421-016-3334-x (2016)

56. Allen D.G., Lamb G.D., Westerblad H. Skeletal muscle fatigue: cellular mechanisms. Physiol Rev. 88, 287-332. https://doi.org/10.1152/physrev.00015.2007 (2008)

57. Allen D.G., Lãnnergren J., Westerblad H. Muscle Cell Function During Prolonged Activity: Cellular Mechanisms of Fatigue. Exp Physiol. 84,97-527.

https://doi.org/10.1113/expphysiol.1995.sp003864 (1995)

58. Favero T.G. Sarcoplasmic reticulum Ca2+ release and muscle fatigue. J Appl Physiol. 87,471-83. https://doi.org/10.1152/jappl.1999.87.2.471 (1999)

59. Westerblad H., Allen D.G. Cellular mechanisms of skeletal muscle fatigue. American Journal of Physiology-Cell Physiology. 261,195-209. https://doi.org/10.1152/ajpcell.1991.261.2.C195 (2009)

60. Fitts R.H. The cross-bridge cycle and skeletal muscle fatigue. J Appl Physiol.104,551-8. https://doi.org/10.1152/japplphysiol.01200.2007 (2008)

61. Bigland-Ritchie B., Johansson R., Lippold O.C., Woods J.J. Contractile speed and EMG changes during fatigue of sustained maximal voluntary contractions. J Neurophysiol. 50,313-24. https://doi.org/10.1152/jn.1983.50.1.313 (1983)

62. Sadoyama T., Miyano H., Higashi Y. Frequency Analysis of Surface EMG to Evaluation of Muscle Fatigue. Eur J Appl Physiol. 47,239-46. https://doi.org/ 10.1007/bf00422469 (1981)

\section{Tables}

Table 1 Characteristics of the subjects in relation to anthropometric data, absolute and predicted values of lung function, respiratory muscle strength and physical activity level 


\begin{tabular}{|c|c|}
\hline Subjects $_{(n)}$ & 22 \\
\hline Age (years) & $24.36 \pm 2.06$ \\
\hline Height $_{(m)}$ & $1.71 \pm 0.08$ \\
\hline Weight $(\mathrm{kg})$ & $65.15 \pm 9.61$ \\
\hline $\operatorname{BMI}\left(\mathrm{kg} / \mathrm{m}^{2}\right)$ & $22.40 \pm 2.02$ \\
\hline $\mathrm{FVC}_{(\mathrm{L})}$ & $4.43 \pm 0.82$ \\
\hline FVC (\%pred) & $95.92 \pm 8.96$ \\
\hline $\mathrm{FEV}_{1(\mathrm{~L})}$ & $3.75 \pm 0.65$ \\
\hline $\mathrm{FEV}_{1 \text { (\%pred) }}$ & $94.54 \pm 7.45$ \\
\hline $\mathrm{FVC} / \mathrm{FEV}_{1}$ & $0.84 \pm 0.047$ \\
\hline FVC/FEV 1 (\%pred) & $96.65 \pm 7.41$ \\
\hline $\mathrm{FEF}_{25-75 \%(\mathrm{~L} / \mathrm{s})}$ & $3.94 \pm 0.7$ \\
\hline $\mathrm{FEF}_{25-75 \%(\mathrm{~L} / \mathrm{s})}$ & $84.7 \pm 13.4$ \\
\hline $\mathrm{MIP}_{(\mathrm{cmH} 2 \mathrm{O})}$ & $106.3 \pm 18.8$ \\
\hline MIP (\%pred) & $113.3 \pm 30.8$ \\
\hline $\mathrm{MEP}_{(\mathrm{cmH} 2 \mathrm{O})}$ & $90.6 \pm 17.8$ \\
\hline MEP (\%pred) & $100.1 \pm 20.2$ \\
\hline SNIP $_{(\mathrm{cmH} 2 \mathrm{O})}$ & $88.0 \pm 15.8$ \\
\hline SNIP $_{(\% p r e d)}$ & $104.5 \pm 31.4$ \\
\hline SNEP $\left._{(\mathrm{cmH}} 20\right)$ & \\
\hline Very active & \\
\hline Active & $\left.\begin{array}{ll}5 & {[22.7 \%}\end{array}\right]$ \\
\hline Irreg. Active A & $10 \quad[45,45 \%]$ \\
\hline Irreg. Active B & 7 [31.8\%] \\
\hline Sedentary & \\
\hline
\end{tabular}


Data presented as mean \pm SD. FVC: forced vital capacity; FEV1: forced expiratory volume in the first second; FVC/FEV1: ratio of forced vital capacity to forced expiratory volume in the first second; FEF2575\%: forced expiratory flow at 25-75\%; MIP: maximum inspiratory pressure; MEP: maximum expiratory pressure; SNIP: sniff nasal inspiratory pressure; SNEP: nasal expiratory pressure; m: meters; kg: kilograms; L: liters; \%pred: percentage of predicted; I/s: liters for second; $\mathrm{cmH} 2 \mathrm{O}$ : centimeters of water Table 2 Parameters related to the performance of the endurance test

\begin{tabular}{|lll|}
\hline & $\mathrm{IPTL}$ & $\mathrm{NH}$ \\
\hline Tlim $(\mathrm{s})$ & $151 \pm 85.7$ & $209.2 \pm 119.6$ \\
Borg $_{\text {initial }}$ & $0.63 \pm 1.13$ & $0.45 \pm 1.0$ \\
Borg $_{\text {Final }}$ & $6.68^{\mathrm{a} *} \pm 1.78$ & $7.13^{\mathrm{a} *} \pm 1.32$ \\
$\mathrm{HR}_{\text {initial }}$ & $81.23 \pm 9.86$ & $76.32 \pm 11.49$ \\
$\mathrm{HR}_{\text {Final }}$ & $104.9^{*} \pm 22.55$ & $104.5^{\star} \pm 21.49$ \\
$\mathrm{SpO}_{2 \text { initial }}(\%)$ & $98 \pm 0.87$ & $98.6 \pm 1.04$ \\
$\mathrm{SpO}_{2 \text { Final }}(\%)$ & $98.3 \pm 1.21$ & $96.7 \pm 3.45$ \\
$\left.\mathrm{Load}_{(\mathrm{cmH}} \mathrm{O}\right)$ & $86.16 \pm 14.88$ & - \\
$\mathrm{VE}(\mathrm{I} / \mathrm{min})$ & - & $104.8 \pm 18.88$ \\
$\mathrm{RR}$ & - & $44.3 \pm 7.79$ \\
\hline
\end{tabular}

Data presented as mean \pm SD. Tlim: time to task failure; HRinitial: pré-test heart rate; HRfinal: pós-test heart rate; Spo2initial: pré-test peripheral arterial saturation; Spo2Final: pós-test peripheral arterial saturation; VE: minute volume; RR: respiratory rate; s: second; \%: percentage; $\mathrm{cmH} 2 \mathrm{O}$ : centimeters of water; I/min: liters for minute. a: nonparametric data analyzed by wilcoxon, but presented on average. *intragroup difference $(p<0,001)$

\section{Figures}



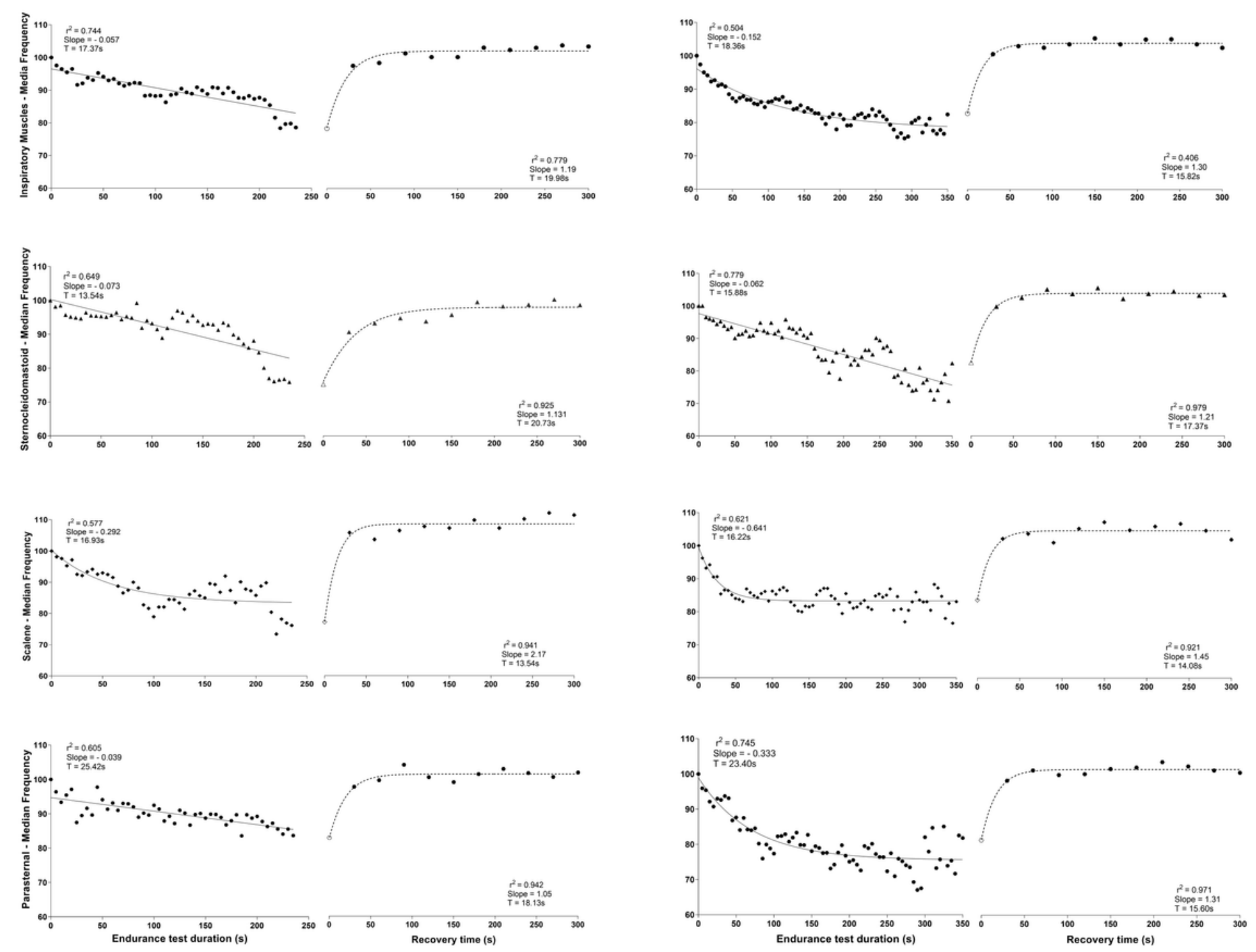

\section{Figure 1}

Time courses of normalized median frequency of the inspiratory ribcage muscles (mean values of the three inspiratory muscles studied) sternocleidomastoid (triangles), scalene (diamonds), parasternal (hexagons), respectively, during the performance of the endurance and recovery test. Each point during the test is the average of 5 seconds; while during recovery each point represents data extracted from an SNIP maneuver. In each muscle, the starting point at time zero (white symbols) corresponds to the last point of the endurance test. 
Inspiratory pressure threshold load
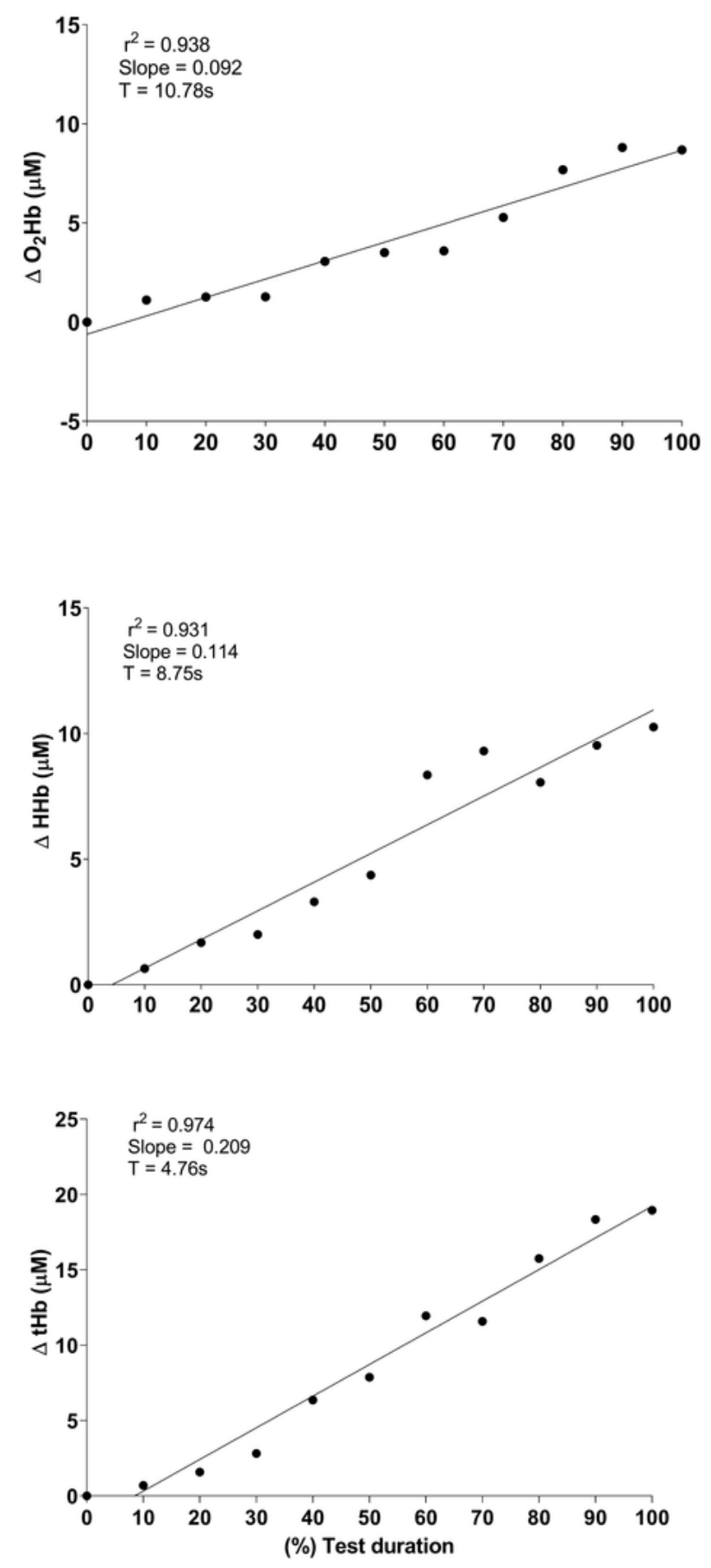

\section{Normocapnic hyperpnea}
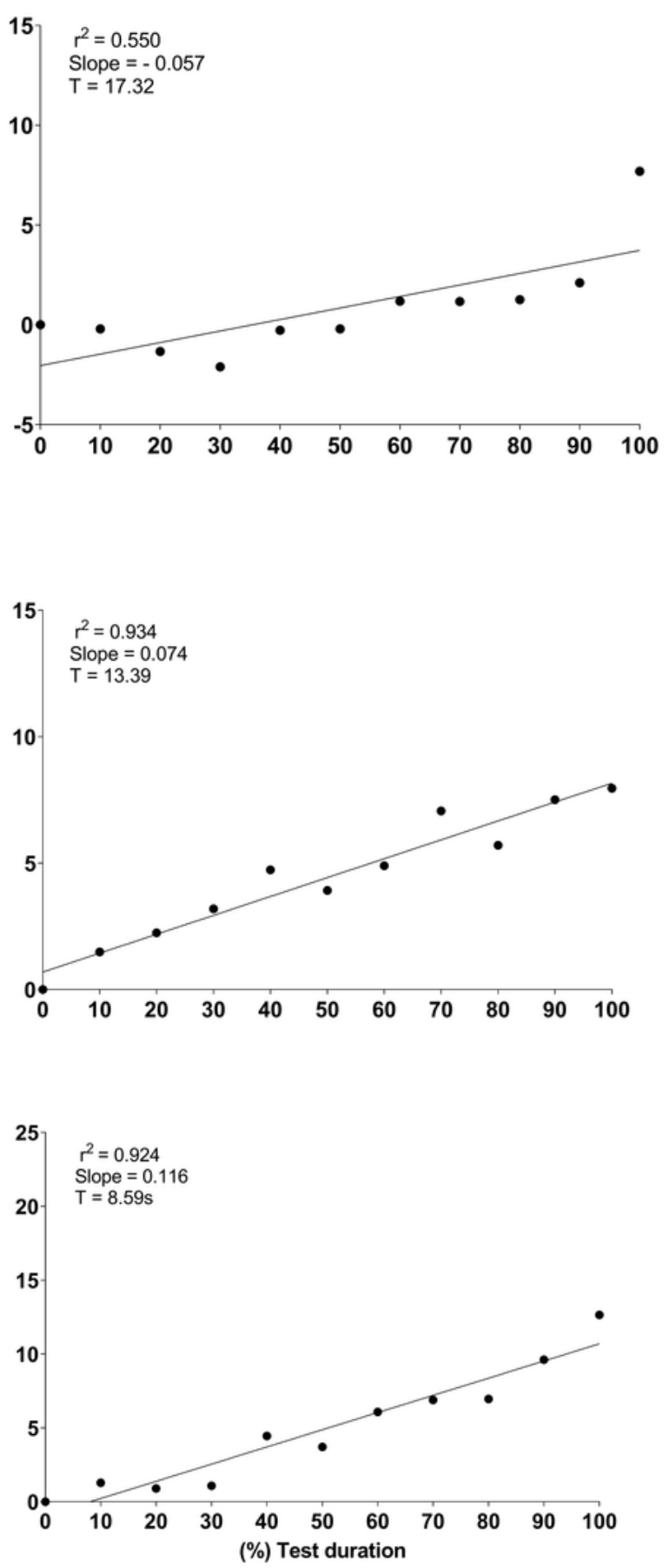

\section{Figure 2}

Time courses of the tissue oxygenation variables of the sternocleidomastoid muscle during the endurance test. Each point during the test is the average of every $10 \%$ of the test duration until the time limit. Abbreviations: $\mathrm{O} 2 \mathrm{Hb}=$ Oxyhemoglobin; $\mathrm{HHb}=$ deoxyhemoglobin; $\mathrm{tHb}^{\prime}=$ total hemoglobin. $\%=$ percentage. 

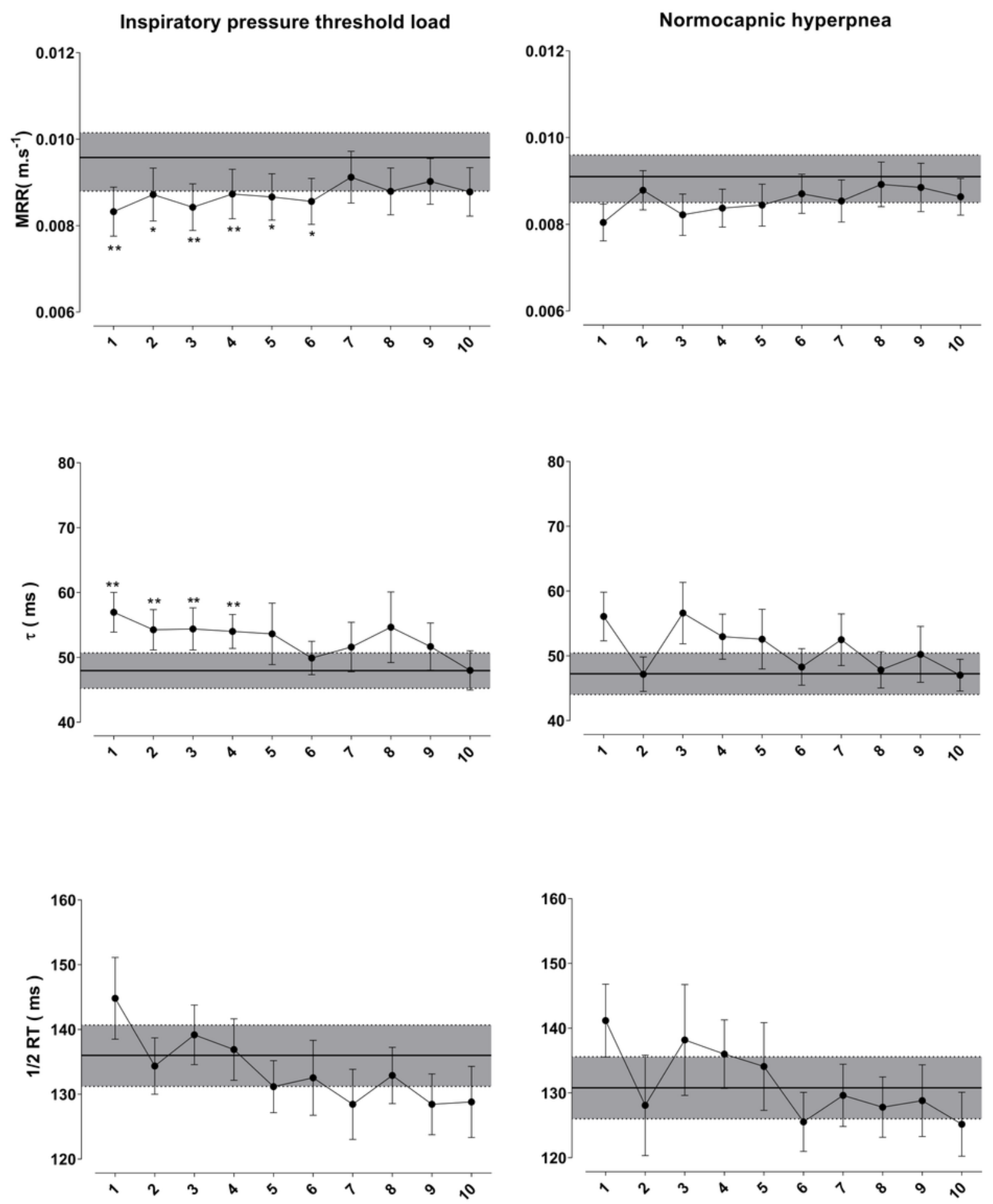

\section{Figure 3}

Variation of relaxation parameters obtained from the sniff traces. Comparison of values during recovery from the endurance test with pre-test values (gray band). Data presented as mean $\pm \mathrm{SE}$. Abbreviations: MRR: maximum relaxation rate; $\tau$ : time constant (tau) of the relaxation curve; $1 / 2 \mathrm{RT}$ time to reach half of the relaxation curve; ms: milliseconds. ${ }^{*} p<0.05 * * p<0.01$. 

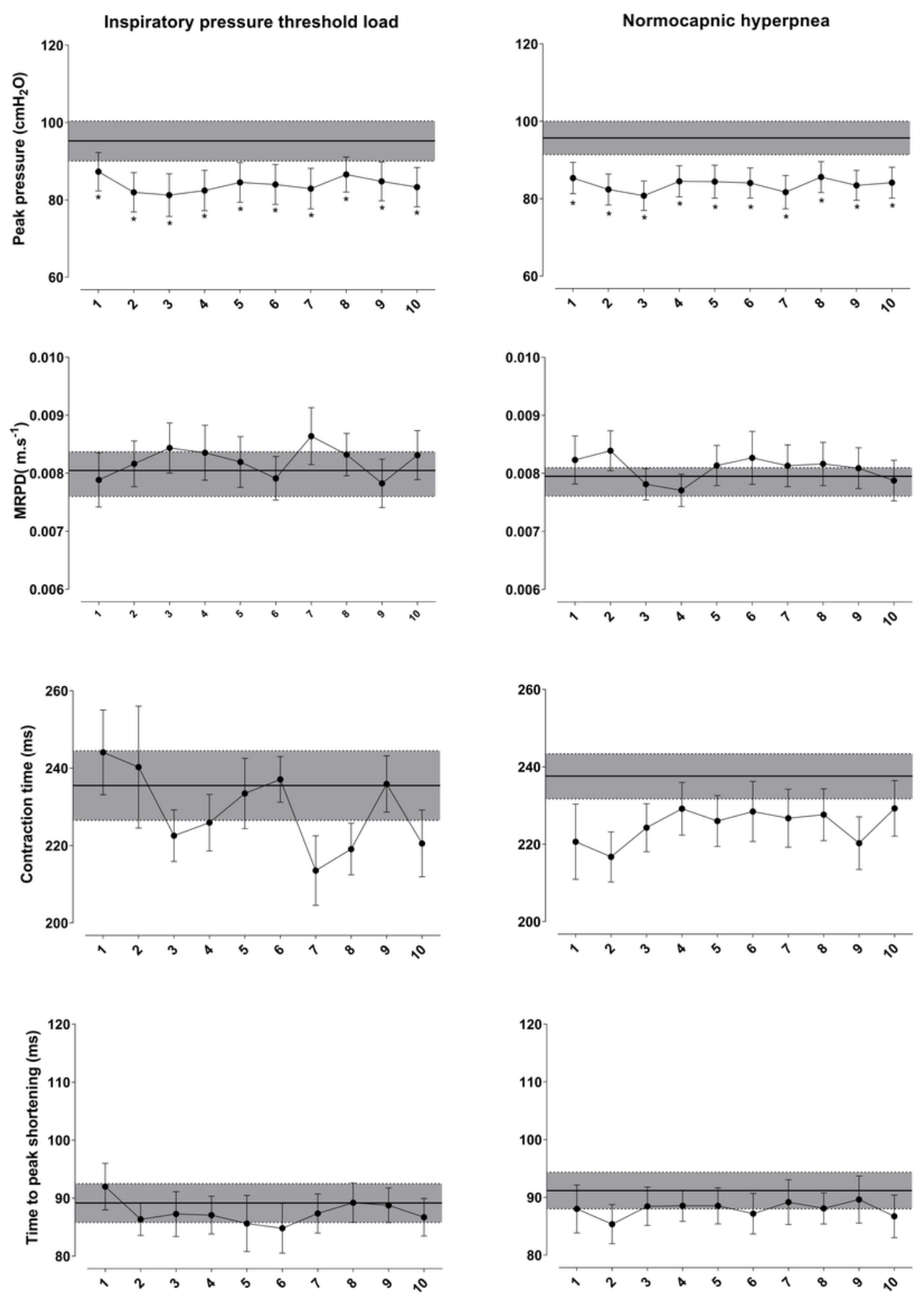

\section{Figure 4}

Variation of the contraction parameters obtained from the sniff traces. Comparison of values during recovery from the endurance test with pre-test values (gray band). Abbreviations: MRPD: maximum rate of pressure development CT: contraction time; TPS: time to reach the MRPD. Data presented as mean \pm SE. MRPD: maximum rate of pressure development; $\mathrm{cmH} 20$ : centimeters per water ms: milliseconds. * $\mathrm{p}$ $<0.05$. 

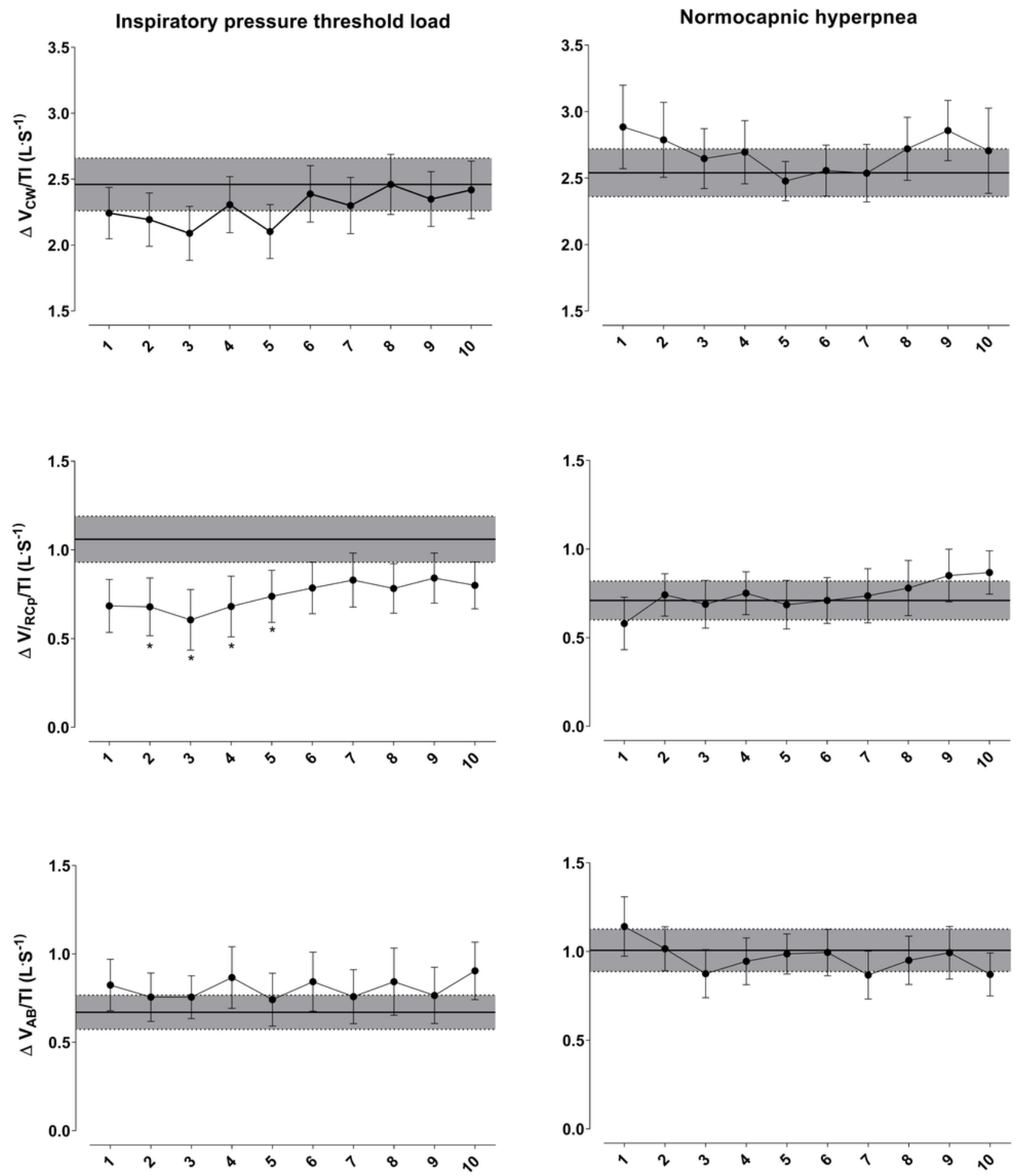

\section{Figure 5}

Variation in the respiratory muscle shortening velocity index. Comparison of the values during the recovery of the endurance test with the pre-test values (gray band). Global inspiratory muscle ( $\triangle \mathrm{VCW} /$ $\mathrm{Ti})$; inspiratory ribcage $(\triangle \mathrm{VRCp} / \mathrm{Ti})$; diaphragm $(\triangle \mathrm{VAB} / \mathrm{Ti})$. Data presented as mean $\pm \mathrm{SE}$. Abbreviations: L.s: liters per second. ${ }^{*} p<0.05$. 

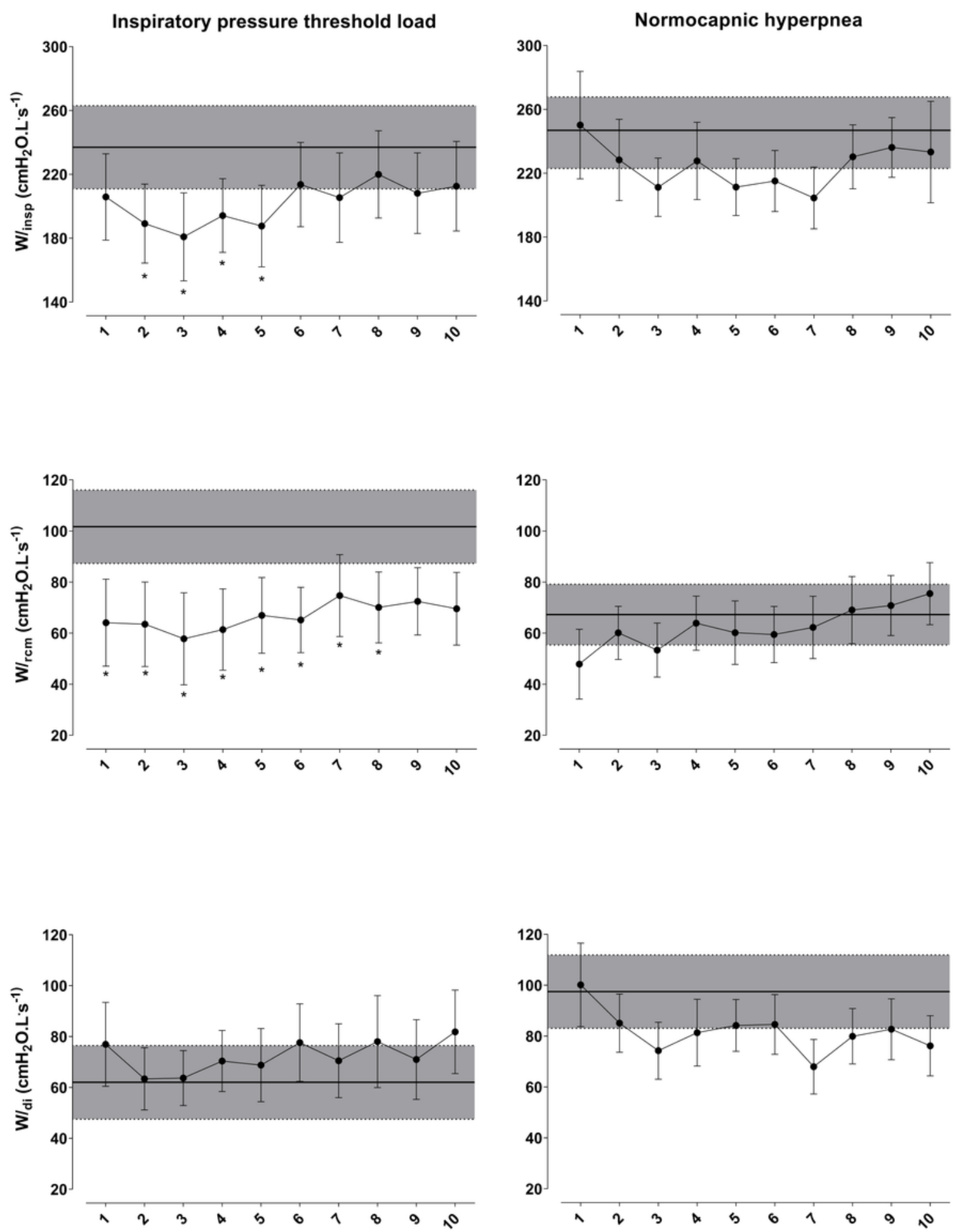

\section{Figure 6}

Variation of the mechanical power of the respiratory muscles. Comparison of the values during the recovery of the endurance test when compared to the pre-test values (gray band). Global inpiratory muscles (Winsp), inspiratory ribcage (Wrcm) and diaphragm (Wdi). Data presented as mean $\pm \mathrm{SE}$. Abbreviations: $\mathrm{cmH} 2 \mathrm{O}$ : water centimeters; L.s: liters per second. ${ }^{*} \mathrm{p}<0.05$. 

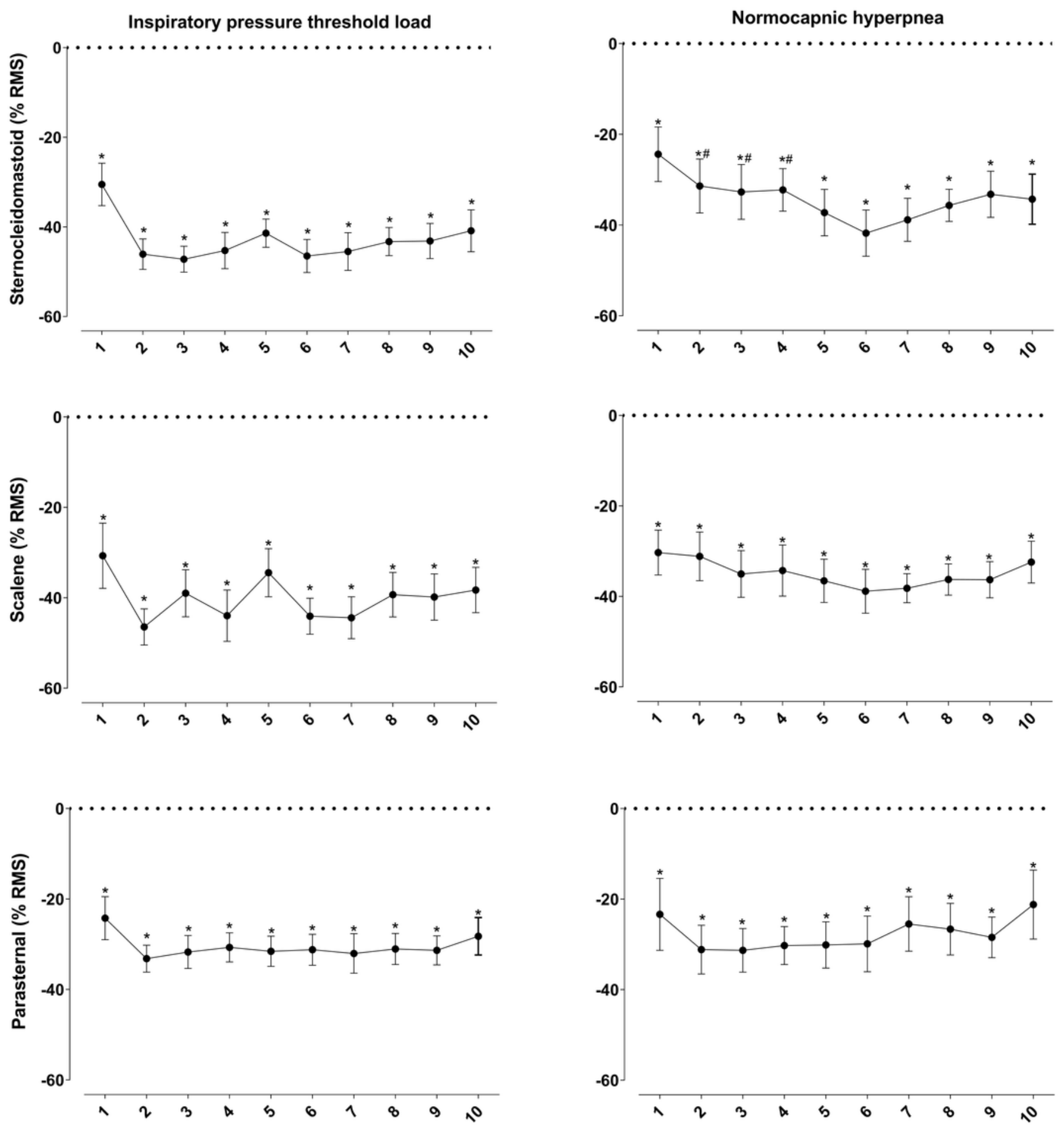

Figure 7

Variation in muscle activation (RMS) of the inspiratory ribcage muscles. Changes from the pre value. Data presented as mean \pm SE. ${ }^{*} p<0.05 \#$ : intergroup difference $(p<0.05)$

\section{Supplementary Files}


This is a list of supplementary files associated with this preprint. Click to download.

- Appendix.docx 